\title{
Short communication: The effect of novel antiseptic compounds on umbilical cord healing and incidence of infection in dairy calves
}

\author{
A. L. Fordyce, ${ }^{1}$ L. L. Timms, K. J. Stalder, and H. D. Tyler \\ Department of Animal Science, lowa State University, Ames 50011
}

\begin{abstract}
The goal of dipping the umbilical cord after birth in calves is to promote healing of the umbilical stump, prevent infection, and encourage the umbilical tissue to detach from the body. Treatment applied to the umbilical area is an important management step for preventing morbidity and mortality in calves. The objective of this study was to compare the effect of 4 umbilical dips on the healing rate, incidence of infection, and age at umbilical cord detachment using newborn Holstein heifer calves $(\mathrm{n}=73)$. Calves were alternately assigned by birth order to 4 treatment groups: $7 \%$ iodine, a dry dip formulated using an antibacterial peptide (nisin) mixed with talc (3.105 g of nisin per $100 \mathrm{~g}$ of talcum powder on a weight per weight basis), liquid nisin (64 $\mu \mathrm{g} / \mathrm{mL}$ ), and $4 \%$ chlorhexidine mixed with alcohol in a 50:50 solution. Umbilical cords were dipped 30 min after birth. Before initial dipping, umbilical cord diameter (as an indicator of the rate of cord drying and healing rate) was determined using a digital caliper. The caliper measurements were repeated at $24 \pm 1,48 \pm 1$, and 72 $\pm 1 \mathrm{~h}$ ( \pm standard deviation) of age and were continued daily until the umbilical cord healed and detached from the animal's body. Diagnosed umbilical infections were documented by veterinary staff based on a combination of clinical symptoms (redness, swelling, purulent discharge, painful response (flinch or kicking) to palpation of the umbilical stump) in addition to a lack of appetite and fever. Data were analyzed using MIXED model procedures with fixed effect of umbilical treatment. No treatment differences were noted between dips on the umbilical cord drying rate or days for umbilical cord to detach. Treatment effects were observed on incidence of umbilical infection (incidence of infection for calves across all treatments was 9.0\%).
\end{abstract}

Key words: antiseptic compound, calf, umbilical cord, umbilical infection

Received May 17, 2017.

Accepted February 17, 2018.

${ }^{1}$ Corresponding author: afordyce@iastate.edu

\section{Short Communication}

The umbilical cord is an important placentally derived structure that maintains the blood supply between the fetus and the placenta throughout pregnancy. The umbilical cord ruptures by way of a tearing process, severing the umbilical artery and vein during the last stage of the birth process, leaving an umbilical stump and umbilical cord remnant on the calf's abdomen. This cord remnant and umbilicus stump, if not disinfected, may become a site for pathogen entry that increases the calf's risk of septicemia. Umbilical infections are not only a health risk for the animal, but also reduce total BW gain during the first 3 mo of the calf's life (Virtala et al., 1996a). Studies indicate that between 5 and $20 \%$ of dairy calves in the United States develop umbilical infections (Virtala et al., 1996b; Mee, 2008 ) and $1.6 \%$ of reported calf deaths are related to umbilical infections (USDA, 2010).

Typically, antiseptic compounds are used to help clean, sanitize, and improve the rate of umbilical stump healing while reducing the risk of infection for the animal. The 2 most common antiseptic compounds used in the dairy industry are $7 \%$ iodine or $4 \%$ chlorhexidine (Mee, 2008). Careful and routine umbilical cord care with an antiseptic and keeping the calf in a clean, dry, well-ventilated area can substantially decrease calf mortality and morbidity (Mee, 2008).

Iodine is produced and commercially available in many concentrations. Seven percent iodine concentration is strong enough to kill most pathogens with a short contact time, and alcohol has been reported to assist in an increased umbilical stump desiccation rate (Imdad et al., 2013). Iodine is bactericidal, sporicidal, cysticidal, and virucidal (WHO, 1999). However, the sale, shipping, and storage of $7 \%$ iodine solutions has become more challenging in recent years due to increased federal regulations (US Department of Justice, 2007), which has created the need to explore antiseptic alternatives.

Chlorhexidine is a good alternative antiseptic for producers due to the broad spectrum of activity against both gram-positive and gram-negative bacteria; in ad- 
dition, it has a relatively long duration of activity, low toxicity, and typically does not irritate skin to the same extent as iodine (Imdad et al., 2013; Sinha et al., 2015). Previous literature in human infants provides consistent evidence that $4 \%$ chlorhexidine can reduce both umbilical cord and periumbilical infection risk (Mullany et al., 2003). To decrease costs, some producers will mix alcohol with chlorhexidine. However, alcohol does not promote drying, is less effective against bacteria than other antimicrobials, and delays cord separation due to tissue damage (WHO, 1999).

Nisin is a natural antimicrobial peptide that was isolated from Lactococcus lactis in 1947 and is the oldest known lantibiotic (Mattick and Hirsch, 1947). It is a nontoxic polypeptide with antimicrobial properties (Tong et al., 2014). Potentially, nisin is a safe alternative to more traditional chemical germicides, such as iodines and chlorhexidines, and is now being explored for other medical usages. Nisin possesses antibacterial activity against a broad range of gram-positive bacteria and a small number of gram-negative bacteria (Tong et al., 2014). Sears et al. (1992) showed a nisin-based formulation used as a postmilking teat dip decreased mastitis and showed a mean log reduction of 3.90 against $S$. aureus and $4.22 \log$ reduction against $E$. coli after a $1 \mathrm{~min}$ exposure to the germicide. Currently, nisin is widely used as an antimicrobial peptide in food preservation (Tong et al., 2014), as well as the primary ingredient in a cow teat wipe used to clean and sanitize the teat area before and after milking (Immucell Corp., Portland, ME). This commercial teat wipe product uses nisin to dramatically reduce levels of common mastitis pathogens, including Staphylococcus aureus, Streptococcus agalactiae, Escherichia coli, Streptococcus uberis, and Klebsiella pneumoniae (Broadbent et al., 1989). The scientific literature on the most common bacteria causing umbilical infections in animals is very limited; however, group B Streptococcus organisms, $S$. aureus, and $E$. coli are the primary pathogens that cause umbilical infections in infants (Mullany et al., 2003). These data suggest that gram-positive bacteria normally present in feces can significantly contribute to the risk of umbilical infections. Nisin can be incorporated in both dry umbilical antiseptic formulations (created by mixing the compound with talcum powder to potentially increase the umbilical tissue drying and healing rate) as well as in a liquid form, similar to other traditional antiseptics.

The purpose of this study was to address the need in the industry to provide producers with an alternative to iodine and chlorhexidine. To accomplish this objective, the effect of 4 umbilical dips on the healing rate, incidence of infection, and age at umbilical cord detach- ment using newborn Holstein heifer calves $(\mathrm{n}=73)$ was compared. The number of calves enrolled in this study was limited to the number of heifer calves born within the defined study time frame $(6 \mathrm{mo})$.

The Iowa State University Animal Care and Use Committee approved this project (log \#10-14-7881B). Holstein heifer calves $(\mathrm{n}=73)$ were enrolled in this trial and monitored at the Iowa State University Dairy Teaching and Research Facility (Iowa State University, Ames) during June through November 2015. All heifer calves were moved within the first hour after birth from the maternity pen to an individual $1.2 \mathrm{~m} \times 1.8 \mathrm{~m}$ calf pen that was bedded with straw within a 96-stall, 4-row indoor calf facility. Calves were alternately assigned by birth order to 4 treatment groups: $7 \%$ iodine (I), a dry dip formulated using an antibacterial peptide (nisin) mixed with talc $(3.105 \mathrm{~g}$ of nisin per $100 \mathrm{~g}$ of talcum powder on a weight per weight basis; $\mathbf{D N}$ ), liquid nisin $(64 \mu \mathrm{g} / \mathrm{mL} ; \mathbf{L N})$, and $4 \%$ chlorhexidine mixed with alcohol in a 50:50 solution (CH). Due to the farm's accepted standards for minimum care, no negative control was used in this trial. Before initial umbilical cord dipping, umbilical cord diameter (an indicator of cord desiccation rate and healing) was measured by trained research personnel using a digital caliper (Mitutoyo 500-197-30 Absolute Digital Caliper, Aurora, IL). This measurement was taken by measuring the width of the umbilical tissue approximately $2.5 \mathrm{~cm}$ below the umbilical stump. Umbilical cords were dipped using approximately $30 \mathrm{~mL}$ of antiseptic solution in a small disposable cup. The umbilical tissue (amnion and umbilical vessels) and umbilical stump (skin, subcutis, and umbilical vessels comprising the portion of the navel cord on the exterior of the abdominal wall) were dipped for approximately $5 \mathrm{~s}$ to ensure the umbilical cord opening, tissue, and umbilical stump were thoroughly covered with the antiseptic solution. The calf was weighed (before feeding colostrum), identification tags were placed in both right and left ears, and pasteurized colostrum was fed within $1 \mathrm{~h}$ of birth before being moved to the individual calf stalls. The digital caliper was used to measure the umbilical diameter repeatedly at approximately $24 \pm 1,48 \pm 1$, and $72 \pm$ $1 \mathrm{~h}$ after birth. Trained members of the research team who were blind to the treatments performed umbilical cord diameter measurements daily until the remaining external umbilical tissue healed to the point of detachment. University staff veterinarians checked on calves every other day during the trial duration and diagnosed the presence of umbilical infection based on umbilicus stump condition [redness, swelling, painful response to palpation (flinch or kicking), and purulent discharge] and calf health (lack of appetite and fever). Calf age 
Table 1. Effect of 7\% iodine, dry nisin, liquid nisin, and 50:50 chlorhexidine:alcohol treatments on umbilical parameters (results presented as $\mathrm{LSM} \pm \mathrm{SE}$ unless otherwise noted) in 73 Holstein calves

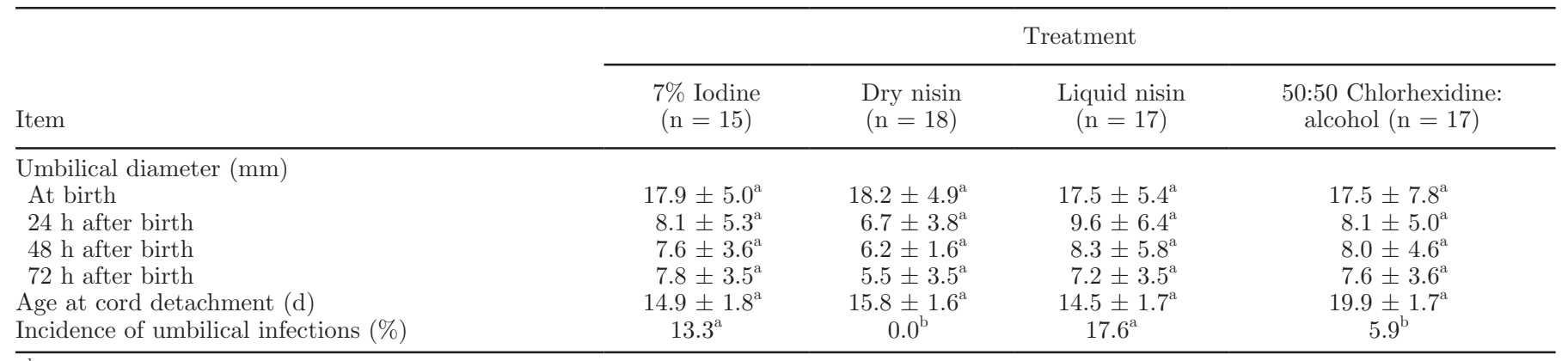

${ }^{\mathrm{a}, \mathrm{b}}$ Means within a row with different superscripts differ $(P<0.05)$.

when umbilical tissue (amnion and umbilical vessels) detached was also recorded, and observations for each calf ended at the time of umbilical cord detachment.

Calves exited the study if they were too weak to stand unassisted. Based on this criterion, 4 heifer calves were excluded due to severe dehydration rendering them too weak to stand. Three of the 4 calves were within the I treatment group and 1 of the 4 calves were within the LN treatment group. Additionally, 2 heifer calves died due to unrelated conditions (the first was born with atresia coli and the second developed septicemia resulting from traumatic injury) during the study period and data from these calves were excluded from the final analysis. Therefore, 67 heifer calves were included in the final statistical analysis. All data were analyzed using MIXED model procedures (PROC MIXED, SAS version 9.2, SAS Institute Inc., Cary, NC). All models included the fixed effect of umbilical treatment. Umbilical diameter at birth was included as a linear covariate in the model used to analyze these data. The quadratic form for umbilical diameter covariate was included in initial models and was not significant and thus was not included in the final analysis models. The random statement included calf number. The covariance structure for the repeated variable was auto-regressive (AR). Degrees of freedom for $t$-tests were adjusted using the Kenward-Roger option in SAS. Fixed effect interactions were not included in the statistical model due to the relatively small sample size. Significance was declared at $P \leq 0.05$. When model effects were significant, treatment means were separated using the pdiff option in SAS. Additionally, a noninferiority analysis was performed on these data using PROC PLM and the appropriate contrast statements.

No treatment differences were noted $(P=0.22)$ between umbilical dips on umbilical cord healing rate as determined by 24 -h umbilical diameter change. The least squares mean and standard error for diameter at birth for calves in treatment group I was $17.9 \pm$
$5.0 \mathrm{~mm}$ and healed to a mean diameter of $8.1 \pm 5.3$ $\mathrm{mm}$ (Table 1). Mean diameter at birth for calves in treatment group DN was $18.2 \pm 4.9 \mathrm{~mm}$ and healed to a mean diameter of $6.7 \pm 3.8 \mathrm{~mm}$ (Table 1). Mean diameter at birth for calves in treatment group LN was $17.5 \pm 5.4 \mathrm{~mm}$ and healed to a mean diameter of $9.6 \pm$ $6.4 \mathrm{~mm}$ (Table 1). Mean diameter at birth for calves in treatment group $\mathrm{CH}$ was $17.5 \pm 7.8 \mathrm{~mm}$ and healed to a mean diameter of $8.1 \pm 5.0 \mathrm{~mm}$ (Table 1).

Mean umbilical cord diameter for all calves across treatments was $17.8 \pm 5.7 \mathrm{~mm}$ at birth and cords healed to a mean diameter of $8.1 \pm 5.0 \mathrm{~mm}$ at $24 \mathrm{~h}$ after birth. Umbilical cord diameter at birth significantly influenced the umbilical diameter at 24,48 , and $72 \mathrm{~h}$ after birth $(P=0.0003)$. However, there was no treatment difference noted for the change in umbilical diameter at the 48 or $72 \mathrm{~h}$ time point $(P=0.25$ and $P=0.13$, respectively). The noninferiority analysis determined that the DN treatment was not inferior to any of the other 3 treatment groups $(P<0.001$ for all comparisons) when the noninferiority margin was set to $2 \mathrm{~mm}$.

Mean age for all calves across treatments when the umbilical tissue detached was $16.3 \pm 7.0 \mathrm{~d}$. Umbilical cord diameter at birth did not affect the calf age when the umbilical cord detachment occurred $(P=0.34)$. Although the effect of treatment on the age of the calf when umbilical cord detachment occurred was not statistically significant $(P=0.06)$, calves dipped with chlorhexidine mixed with alcohol (50:50) detached at an average of $20 \mathrm{~d}$ compared with $15.5 \mathrm{~d}$ for the other 3 treatments $(P=0.05$; Table 1$)$. The noninferiority analysis determined that the DN treatment was not inferior to any of the other 3 treatment groups $(P<$ 0.05 for all comparisons) when the noninferiority margin was set to $5 \mathrm{~d}$.

The overall incidence of umbilical infection across treatments was $9.0 \%$ (6/67 calves diagnosed). The incidence of umbilical infections was greater for calves 
receiving I (13.3\%) and $\mathrm{LN}(17.6 \%)$ treatments when compared with calves receiving DN treatments $(0.0 \%)$ and $\mathrm{CH}(5.9 \%)$ treatments $(P=0.04$; Table 1$)$. The noninferiority analysis determined that the DN treatment was not inferior to any of the other 3 treatment groups $(P<0.05)$ when the noninferiority margin is set to $5 \%$.

Data from this study are consistent with previous findings that evaluated antiseptics and compared umbilical treatment alternatives to $7 \%$ iodine when dipping umbilical cords of newborn calves (Grover and Godden, 2011; Robinson et al., 2015; Wieland et al., 2017). However, the present study did not use a true negative control in which some calves' umbilical cords were not treated with a dip at birth, in contrast to the study performed by Grover and Godden (2011).

The primary objective of this study was to evaluate the efficacy of nisin as an alternative to iodine as an antiseptic suitable for umbilical treatment. The nisinbased formulation showed little or no potential irritation after multiple skin applications, unlike the iodophor and chlorhexidine digluconate teat dips that showed significant skin irritation potential, which is an important property to consider for umbilical treatments. Nisin has never been evaluated as an umbilical dip using the wet or dry formulation before this study. The dry dip formulation used in the current study (freeze-dried liquid nisin mixed with talcum powder) was formulated to theoretically promote desiccation of the umbilicus tissue to allow healing at a faster rate, although no such statistical differences were documented in this study. Potential interactions between bedding type, bedding dryness, and treatment were not measured in this study and may have affected the responses observed.

The reduced effectiveness of the chlorhexidine mixed with alcohol (50:50) on age at umbilical detachment may have been due to the high concentration of alcohol in this treatment. Undiluted chlorhexidine has been reported to be effective as an umbilical dip in past studies. One observational study reported that the risk of calf mortality was significantly reduced when umbilical cords were treated with chlorhexidine, compared with untreated calves (Waltner-Toews et al., 1986). Additionally, in their study, treatment with iodine tended to increase the risk of mortality. However, these results could potentially be affected by unmeasured confounding factors because many factors are documented to affect the risk of calf mortality (e.g., level of passive immunity, stress at calving, and seasonal effects) that were not measured in this study. Umbilical cord bacterial growth has been documented to be reduced in foals by treatment with $7 \%$ iodine or chlorhexidine compared with untreated cords (Lavan et al., 1994). However, the use of alcohol as an umbilical treatment has not been effective. Golshan and Hossein (2013) reported that in a rural community in Iran, treating the umbilical cord of newborn babies with alcohol (70\% ethanol) delayed umbilical cord separation. Furthermore, in some infants where alcohol was used to clean the umbilical cord for more than $21 \mathrm{~d}$, toxicity symptoms such as hemorrhage, skin necrosis, impaired central nervous system, and hypoglycemia can occur (Golshan and Hossein, 2013). These findings further support the conclusion that alcohol may be detrimental to umbilical cord healing and separation.

Incidence of umbilical infection for all calves in this study was $9.0 \%$, which is much lower than the $27 \%$ incidence of infection reported by Wieland et al. (2017). However, Robinson et al. (2015) reported 0\% incidence of infection in calves under well-ventilated, clean, and dry bedding conditions when calves were monitored for the first $48 \mathrm{~h}$ of life. The incidence of infection in the present study was different across treatments. The findings from this work when combined with previously reported results suggest that antiseptics applied to the umbilical cord after birth may not be able to overcome heavy pathogen loads found in contaminated bedding in the calf pen and maternity area. Previous research has demonstrated that simple iodine tinctures dissolved in alcohol, despite being bactericidal, sporicidal, virucidal, and fungicidal, require a prolonged contact time (Gamage, 2003). In addition, the disinfectant properties of iodine are neutralized in the presence of organic material. Therefore, frequent applications may be needed to thoroughly disinfect the area (Gamage, 2003). Calf pen management, microenvironment, contamination level, and bedding substrate can all affect the effectiveness of most umbilical antiseptic treatments. The effects of these parameters have not been investigated or taken into consideration in the present study or in previous studies with dairy calves (Grover and Godden, 2011; Robinson et al., 2015; Wieland et al., 2017).

In summary, the dry form nisin with talcum powder dip (which mimics dry cord care in humans) is a good alternative to $7 \%$ iodine, but is not currently commercially available to the US livestock industry. It has become more difficult to obtain and store $7 \%$ iodine solutions in large quantities. Although $2 \%$ iodine solutions are not regulated and continue to be commercially available, $7 \%$ iodine solutions are 3 times more concentrated and more effective in keeping the umbilical area disinfected in contaminated environments compared with $2 \%$ iodine and should be preferred. Chlorhexidine products that do not contain alcohol are a good alternative to iodine; however, the relatively high cost can be a significant economic issue for dairy producers. In conclusion, dips evaluated in this trial performed similarly for most measured parameters; however, calves 
receiving I and LN treatments experienced an increased incidence of umbilical infection compared with calves receiving $\mathrm{DN}$ and $\mathrm{CH}$ treatments under the conditions of this trial.

\section{ACKNOWLEDGMENTS}

The authors acknowledge Immucell Corporation (Portland, ME) for their support for this trial.

\section{REFERENCES}

Broadbent, J. R., Y. C. Chou, K. Gillies, and J. K. Kondo. 1989 Nisin inhibits several gram-positive, mastitis-causing pathogens. J. Dairy Sci. 72:3342-3345. https://doi.org/10.3168/jds.S0022 -0302(89)79496-0.

Gamage, B. 2003. A guide to selection and use of disinfectants. Accessed Mar. 19, 2017. http://www.mtpinnacle.com/pdfs/ disinfectant-selection-guidelines.pdf.

Golshan, M., and N. Hossein. 2013. Impact of ethanol, dry care and human milk on the time for umbilical cord separation. J. Pak. Med. Assoc. 63:1117-1119.

Grover, W. M., and S. Godden. 2011. Efficacy of a new navel dip to prevent umbilical infection in dairy calves. Bov. Pract. 45:70-77.

Imdad, A., R. M. M. Bautista, K. A. A. Senen, M. Uy, V. Esterlita, I. Mantaring, B. Jacinto, and Z. A. Bhutta. 2013. Umbilical cord antiseptics for preventing sepsis and death among newborns. Cochrane Database of Systematic Reviews. 5. John Wiley \& Sons, Ltd. http://onlinelibrary.wiley.com/doi/10.1002/14651858 CD008635.pub2/abstract

Lavan, R. P., J. Madigan, R. Walker, and N. Muller. 1994. Effects of disinfectant treatments on the bacterial flora of the umbilicus of neonatal foals. Pages 37-38 in Proceedings of the 40th Annual Convention of the American Association of Equine Practitioners, Vancouver, BC, Canada. American Association of Equine, Lexington, $\mathrm{KY}$.

Mattick, A. T., and A. Hirsch. 1947. Further observations on an inhibitory substance (nisin) from lactic streptococci. Lancet 2:5-8.

Mee, J. F. 2008. Managing the calf at calving time. Am. Assoc. Bov. Pract. Proc. 41:46-53.

Mullany, L. C., G. L. Darmstadt, and J. M. Tielsch. 2003. Role of antimicrobial applications to the umbilical cord in neonates to pre- vent bacterial colonization and infection: A review of the evidence. Pediatr. Infect. Dis. J. 22:996-1002.

Robinson, A. L., L. L. Timms, K. J. Stalder, and H. D. Tyler. 2015. The effect of four antiseptic compounds on umbilical cord healing and infection rates in the first $24 \mathrm{~h}$ in dairy calves from a commercial herd. J. Dairy Sci. 98:5726-5728. https://doi.org/10.3168/ jds.2014-9235.

Sears, P. M., B. S. Smith, W. K. Stewart, R. N. Gonzalez, S. D. Rubino, S. A. Gusik, E. S. Kulisek, S. J. Projan, and P. Blackburn. 1992. Evaluation of nisin-based germicidal formulation on teat skin of live cows. J. Dairy Sci. 75:3185-3190. https://doi.org/10 .3168/jds.S0022-0302(92)78083-7.

Sinha, A., S. Sazawal, A. Pradhan. S. Ramji, and N. Opiyo. 2015 Chlorhexidine skin or cord care for prevention of mortality and infections in neonates. Cochrane Database Syst. Rev. CD007835 https://doi.org/10.1002/14651858.CD007835.pub2.

Tong, Z., L. Ni, and J. Ling. 2014. Antibacterial peptide nisin: A potential role in the inhibition of oral pathogenic bacteria. Peptides 60:32-40. https://doi.org/10.1016/j.peptides.2014.07.020.

USDA. 2010. Dairy 2007: Heifer Calf Health and Management Practices on U.S. Dairy Operations. USDA, Washington, DC.

US Department of Justice. 2007. Changes in the regulation of iodine crystals and chemical mixtures containing over 2.2 percent iodine. Fed. Regist. 72:35920-35931.

Virtala, A. M. K., G. D. Mechor, Y. T. Grohn, and H. N. Erb. 1996a. The effect of calfhood diseases on growth of female dairy calves during the first 3 months of life in New York state. J. Dairy Sci 79:1040-1049. https://doi.org/10.3168/jds.S0022-0302(96)76457 $-3$.

Virtala, A. M. K., G. D. Mechor, Y. T. Grohn, and H. N. Erb. 1996b. Morbidity from nonrespiratory diseases and mortality in dairy heifers during the first three months of life. J. Am. Vet. Med. Assoc. 208:2043-2046.

Waltner-Toews, D., S. W. Martin, and A. H. Meek. 1986. Dairy calf management, morbidity and mortality in Ontario Holstein herds. IV. Association of management with mortality. Prev. Vet. Med. 4:159-171.

Wieland, M., S. Mann, C. L. Guard, and D. V. Nydam. 2017. The influence of 3 different navel dips on calf health, growth performance, and umbilical infection assessed by clinical and ultrasonographic examination. J. Dairy Sci. 100:513-524. https://doi.org/ $10.3168 /$ jds.2016-11654.

WHO (World Health Organization). 1999. Care of the Umbilical Cord A review of the evidence. Reproductive Health (Technical Support). Maternal and Newborn Health/Safe Motherhood, Geneva, Switzerland. 